\title{
Giant left atrial appendage aneurysm compressing the left ventricular wall diagnosed by multiple imaging technology
}

\author{
Meng Li, Yonghuai Wang, Shuang Liu, Jun Yang, Chunyan Ma \\ Department of Cardiovascular Ultrasound, The First Hospital of China \\ Medical University, Shenyang, Liaoning, People's Republic of China
}

A 46-year-old male presented to the documented hospital having had palpitation over a 2 month period. Electrocardiogram showed atrial fibrillation with a heart rate of approximately $100 \mathrm{bpm}$. He had a history of an atrial septal defect and underwent an atrioseptopexy 23 years prior.

Transthoracic echocardiography showed dilated left and right atria, mild mitral and tricuspid regurgitation, and normal left ventricular ejection fraction (56\%). Furthermore, there was a giant left atrial appendage aneurysm (LAAA), $62 \times 31 \mathrm{~mm}$ in size, compressing the left ventricular wall (Fig. 1A). Doppler imaging showed that blood communicated between the left atrium and LAAA (Fig. 1A), with slightly impaired left atrial appendage function (Fig. 1B). Three-dimensional echocardiography showed distinct pectinate muscles (Fig. 1C, white arrow) and no thrombus in the LAAA (Fig. 1C). Based on speckle tracking imaging, left ventricular global longitudinal strain was $-14.8 \%$, and the bull's eye showed paradoxical movement of compressed basal and mid segments of the anterior, anterolateral, and inferolateral walls (Fig. 1D). Three-dimensional computed tomography reconstruction delineated the appearance of the LAAA (Fig. 1E).

Giant LAAA is a rare congenital or acquired anomaly; the most common cause is congenital, which may relate to maldevelopment of the pectinate muscles. The acquired forms usually result from mitral valve disease. In the present case, although the giant aneurysm was not noted in the previous surgery, it was believed that the LAAA was congenital because the appendage might have grown with age and no mitral valve disease existed. The patient declined surgery despite the high risk of thromboembolism. Drug therapy and regular follow-up care were advised.

Conflict of interest: None declared

Address for correspondence: Chunyan Ma, MD, PhD, First Hospital of China Medical University, No. 155 Nanjingbei Street, Shenyang, Liaoning 110001, China, tel/fax: +86-24-83282114, e-mail: cmu1h_mcy@126.com 


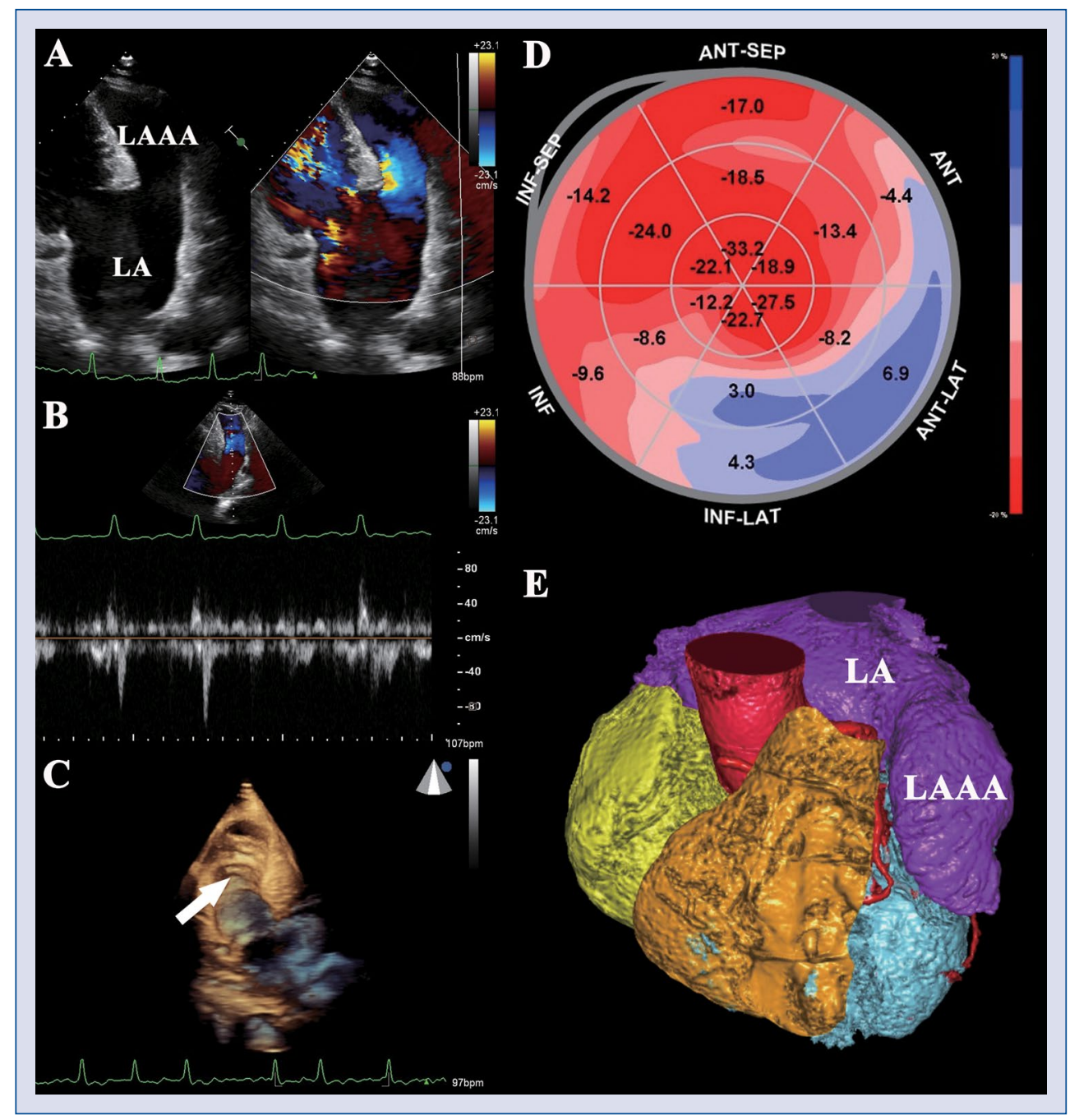

Figure 1. A. Appendage aneurysm showed by transthoracic echocardiography; B. Blood velocity of left atrial appendage; C. Three-dimensional echocardiography showed pectinate muscles (marked by a white arrow) inside the left atrial appendage; D. Global longitudinal strain of the left ventricle wall; E. Three-dimensional computed tomography showed the overall view of the heart; LA — left atrium; LAAA — left atrial appendage aneurysm. 